\title{
QUESTÃO TERRITORIAL, PROCESSO DE REGIONALIZAÇÃO DO SUS E FINANCIAMENTO DAS REDES TEMÁTICAS
}

\section{Territorial approach, process of SUS regionalization and financing of the thematic networks}

\section{Cuestión de territorio, proceso de regionalización del SUS y financiación de las redes temáticas}

\author{
Ligia Schiavon Duarte (Lattes) \\ Instituto de Saúde da Secretaria de Estado da Saúde de São Paulo - IS/SES/SP - São Paulo (SP) - Brasil
}

Áquilas Nogueira Mendes (Lattes)

Faculdade de Saúde Pública - FSP/USP - São Paulo (SP) - Brasil

Pontifícia Universidade Católica de São Paulo - PUC/SP - São Paulo (SP) - Brasil

\section{RESUMO}

Objetivo: Sistematizar as principais características das portarias que orientam a transferências de recursos para a rede de cuidado obstétrico e neonatal (Rede Cegonha), a Rede de Atenção à Urgência (RAU), a Rede de Atenção Psicossocial (RAPS) e a Rede de Atenção à Pessoa com Deficiências (Viver sem limites). Métodos: As portarias foram analisadas a partir de três eixos estruturais: i) perfil assistencial dos elementos financiados, sendo observados os componentes e os equipamentos, insumos e ações; ii) o montante de recursos financeiros disponível para cada um dos elementos financiados, discriminado em investimentos e custeio; e iii) o comprometimento de cada um dos entes federativos no financiamento e execução do recurso. Resultados: A análise permite evidenciar três principais aspectos no repasse de recursos financeiros federais para os entes subnacionais: i) preponderância de recursos para o cuidado especializado, cuja configuração produtiva exige economia de escala, tendendo a se concentrar em municípios que apresentam maior centralidade; ii) indução ao comprometimento de recursos, financeiros ou não, dos entes subnacionais; e iii) grande fragmentação nos repasses financeiros. Conclusão: O financiamento das redes temáticas, além de favorecer o modelo médico assistencial por meio da transferência de recurso prioritariamente para a atenção à saúde voltada à média e alta complexidade, parece tender às regiões privilegiadas.

Descritores: Regionalização; Economia da Saúde; Financiamento da Saúde.

\section{ABSTRACT}

Objective: To systematize the main characteristics of the ordinances that guide the transfer of resources to the obstetric and neonatal care network (Stork Network), the Emergency Care Network (UCA), the Psychosocial Care Network (RAPS), and the Care Network for Persons with Disabilities (Living without limits). Methods: The ordinances were analyzed from three structural axes: i) assistance profile of the financed elements, with observation of the components and equipment, inputs and actions; ii) the amount of financial resources available for each of the financed elements, broken down into investments and operating costs; and iii) the accountability of each of the federative entities in the financing and execution of the resource. Results: The analysis allows the identification of three main aspects in the transfer of federal financial resources to subnational entities: i) preponderance of resources for specialized care, whose productive configuration requires economies of scale, with a tendency to focus on municipalities that are more central; ii) induction to compromising the resources, whether financial or not, of the subnational entities; and iii) great fragmentation in financial transferring. Conclusion: The financing of the thematic networks, in addition to favoring the medical model of health care through the priority transfer of resources to medium-and high-complexity health care, seems to tend to the privileged regions.

Descriptors: Regional Health Planning; Health Economics; Healthcare Financing.

Este artigo foi selecionado, corrigido e aprovado pela Comissão Científica do Fórum Internacional de Sistemas Universais de Saúde, seguindo suas normas e formatação. 


\section{RESUMEN}

Objetivo: Sistematizar las principales características de los documentos que orientan las transferencias de recursos para la red de cuidado obstétrico y neonatal (Red Cigüeña), la Red de Atención de Urgencia (RAU), la Red de Atención Psicosocial (RAPS) y la Red de Atención de la Persona con Discapacidades (Vivir sin límites). Métodos: Se analizaron los documentos a partir de tres ejes estructurales: i) el perfil asistencial de los elementos financiados observando los componentes y los equipos, los insumos y las acciones; ii) la cantidad de recursos financieros disponible para cada uno de los elementos financiados, discriminado en investimentos y coste; y iii) el comprometimiento de cada uno de los entes federativos para la financiación y la ejecución del recurso. Resultados: El análisis permite evidenciar tres aspectos principales en el repase de los recursos financieros federales para los entes subnacionales: i) la preponderancia de recursos para el cuidado especializado cuya configuración productiva exige una economía de escala con propensión para concentrarse en los municipios que presentan mayor centralidad; ii) la inducción al comprometimiento de recursos financieros u no de los entes subnacionales; y iii) la gran fragmentación de los repases financieros. Conclusión: La financiación de las redes temáticas además de favorecer el modelo médico de atención a través de la transferencia de recurso con prioridad para la atención de la salud de media y alta complejidad tiene propensión para las regiones privilegiadas.

Descriptores: Regionalización; Economía de la Salud; Financiación de la Atención de la Salud.

\section{INTRODUÇÃO}

Os esforços recentes no sentido de promover a regionalização da política de saúde têm como perspectiva a consolidação do Sistema Único de Saúde (SUS) como direito social, conforme proposto pela Constituição Federal de 1988. A importância do processo de regionalização do SUS ganha relevância quando se considera que um dos elementos fundantes da Carta Magna é a redução das desigualdades sociais e regionais. Vale destacar que a garantia da saúde como direito social e mitigação das desigualdades são desafios consideráveis diante do turbulento cenário do capitalismo contemporâneo de dominância do capital portador de juros, marcado pela razão neoliberal ${ }^{(1)}$.

A saúde no seu conceito ampliado, tal qual proposto pela Lei $n^{\circ} 8.080$, que define como fatores determinantes e condicionantes da saúde, "entre outros, a alimentação, a moradia, o saneamento básico, o meio ambiente, o trabalho, a renda, a educação, o transporte, o lazer e o acesso aos bens e serviços essenciais" e, ainda, que "os níveis de saúde da população expressam a organização social e econômica do País"(2), evidencia a interdependência entre o setor saúde e os demais setores econômicos e sociais que operam a vida das pessoas. Portanto, o adequado ordenamento territorial, que promova a redução das desigualdades regionais, a melhor distribuição espacial das atividades econômicas, a proteção ambiental e a qualidade de vida da população, é princípio constitucional que deve orientar as políticas públicas de forma geral e a saúde pública em especial(3).

Como é amplamente conhecido, o processo histórico de inserção do Brasil na divisão internacional do trabalho gerou desigualdades territoriais expressas nas diferentes centralidades e hierarquias dos seus municípios ${ }^{(4-6)}$. Conforme aponta Pochmann ${ }^{(6)}$, a dinâmica econômica do país, sobretudo no ciclo de industrialização de 1930-1980, é marcada pela forte tendência à concentração produtiva em poucos e contidos espaços do território nacional. Esse processo, que arrefece a partir da década de 1990, determina a extrema concentração territorial percebida ainda hoje. Apenas como ilustração, verifica-se que, em 2015 , cerca de $40 \%$ do Produto Interno Bruto (PIB) nacional se concentra em apenas 30 dos 5.570 municípios brasileiros ${ }^{(7)}$.

Diante do exposto, é possível afirmar que a regionalização do SUS, inicialmente associada à necessidade de organização das ações e serviços públicos de saúde em uma rede regionalizada e hierarquizada, busca sobretudo alcançar um projeto maior de democratização e mitigação das desigualdades regionais brasileiras.

Nessa perspectiva, espera-se que o processo de regionalização do SUS, fruto da ação do Estado e em coerência com um projeto de ordenamento territorial que se contraponha a tendência de concentração das atividades econômicas determinada pelo mercado na busca da valorização do capital ${ }^{(8)}$, seja orientado para ações que promovam a mitigação dos desequilíbrios regionais.

As Redes de Atenção à Saúde (RAS), que são arranjos organizativos de ações e serviços de saúde ${ }^{(9)}$ ganham protagonismo no processo de regionalização do SUS a partir do Decreto $n^{\circ} 7.508^{(10)}$, no início da década de 2010. Para promover a organização e funcionamento das RAS o governo federal estabeleceu compromissos prioritários por meio das Redes Temáticas de Atenção à Saúde ${ }^{(11)}$. Conforme aponta Magalhães Junior ${ }^{(12)}$, as redes temáticas teriam "capacidade indutora de determinados temas para a organização do conjunto das RAS" (p.19), podendo estar relacionados com a atenção de urgência e emergência, as doenças crônicas no seu conjunto ou em determinados 
grupamentos de patologias, a assistência obstétrica e neonatal e a rede de atenção da saúde mental. Assim, as redes temáticas teriam como perspectiva organizar as RAS a partir do ordenamento dado pela lógica circunscrita a uma (ou mais de uma) linha de cuidado. Haja vista o objetivo final de integralidade da assistência, inicialmente foi planejado induzir linhas de cuidados temáticas que passam a contar com recursos específicos.

Foram definidas as seguintes redes: $i)$ Rede Cegonha ${ }^{(13)}$, que é a rede de cuidado obstétrico e neonatal; ii) Rede de Urgência e Emergência (RUE)(14); iii) Rede de Atenção Psicossocial para pessoas com sofrimento ou transtorno mental e com necessidades decorrentes do uso de crack, álcool e outras drogas (RAPS) $\left.{ }^{(15)} ; i v\right)$ Rede de Cuidados à Pessoa com Deficiências (Viver Sem Limite) ${ }^{(16)}$; v) Rede de Prevenção e Controle do Câncer de Colo do Útero e Mama, depois ampliada para Política Nacional para a Prevenção e Controle do Câncer e inserida como eixo na Rede de Atenção à saúde das pessoas com doenças crônicas ${ }^{(17 .)}$.

Assim, ao se considerar o protagonismo recente que as RAS assumiram no processo de regionalização do SUS ${ }^{(3)}$, torna-se oportuno compreender de que forma as transferências de recursos da esfera federal para os entes subnacionais para as ações e estratégias vinculadas às redes temáticas regionais, que as dinamizam, podem contribuir ou não para a mitigação das concentrações territoriais. Ou seja, compreender os elementos estimuladores ou desestimuladores de determinadas lógicas territoriais no financiamento das redes temáticas regionais possibilita jogar luz sobre o papel do SUS na mitigação, ou não, das desigualdades sociais e, sobretudo, regionais no território nacional.

Com o intuito de ressaltar os elementos que propiciam a maior ou menor concentração territorial na política recente que dinamiza o processo de regionalização do SUS, o presente trabalho tem como objetivo sistematizar as principais características das portarias que orientam a transferências de recursos para a rede de cuidado obstétrico e neonatal (Rede Cegonha), a Rede de Atenção à Urgência (RAU), a Rede de Atenção Psicossocial (RAPS) e a Rede de Atenção à Pessoa com Deficiências (Viver sem limites).

\section{MÉTODOS}

O financiamento das redes temáticas regionais ocorre sobretudo por meio de transferências do Fundo Nacional de Saúde (FNS) para os Fundos Estaduais de Saúde (FES) e Fundos Municipais de Saúde (FMS). Os diferentes componentes que compõem as redes temáticas apresentam diferentes modalidades de financiamento. Além do financiamento feito por meio das transferências do FNS para os FMS e FES, principalmente para custeio de ações e serviços de saúde, mas também para investimentos na construção e reforma de unidades de atendimento e qualificação (compras de equipamentos) dos serviços, foram direcionados recursos para a capacitação de profissionais e gestores, compra e distribuição de materiais, doação de veículos, construções de sistemas de informações, entre outras ações. No entanto, muito embora todas as formas de financiamento devam ser consideradas para a adequada avaliação da implantação das redes temáticas, para os objetivos do presente estudo interessa a parcela de recurso transferida aos entes subnacionais por meio do FNS, por ser essa a principal modalidade.

É possível afirmar que a transferência de recursos das redes temáticas incide de alguma forma sobre três blocos de financiamento, o da Média e alta complexidade ambulatorial e hospitalar (MAC), o de investimento e o da Atenção básica $(A B)$. No entanto, grande parte do montante disponibilizado às estratégias vinculadas às redes temáticas foi direcionada ao bloco MAC ${ }^{3}$.

Com o intuito de apreendermos as principais características do financiamento das redes temáticas foram sistematizadas e organizadas em quadros resumos as portarias de quatro redes temáticas, a saber: a Rede Cegonha (RC), a Rede de Atenção à Urgência (RAU), a Rede de Atenção Psicossocial (RAPS) e a Rede de Cuidados à Pessoa com Deficiências (Viver Sem Limite). Cabe ressaltar, que devido à institucionalização mais recente da Rede de Atenção às Pessoas com Doenças Crônicas (RASPDC) e ao fato de que os incentivos financeiros envolvidos referem-se a diferentes linhas de cuidado específicas, como a relacionada ao cuidado de pessoas com sobrepeso e obesidade ou a de pessoas com doença renal crônica, não foi possível identificar transferências "fundo a fundo" específicas para essa rede temática. Optou-se assim por não considerar tal rede no escopo do presente estudo.

Os quadros resumos foram organizados em três eixos de análise. O eixo que qualifica o perfil assistencial dos elementos financiados, sendo observados os componentes e os equipamentos, insumos e ações. O eixo que dimensiona os recursos disponíveis em valores monetários para cada um dos elementos financiados, discriminado em investimentos e custeio. E, finalmente, o eixo da participação interfederativa, que aponta a participação da União e a contrapartida dos demais entes, descriminando o ente federado, estadual e municipal, com acesso à política. A seguir são apresentados os quatro quadros e a sua análise. 


\section{RESULTADOS E DISCUSSÃO}

É possível observar que dos quatro componentes que compõem a rede, dois não tem financiamento determinado nas portarias da Rede Cegonha - o Puerpério e Atenção Integral à Saúde da Criança e o Sistema Logístico, Transporte Sanitário e Regulação. Já o financiamento previsto para os componentes Pré-natal e Parto e Nascimento são na maior parte direcionados às ações específicas e especializadas.

De forma geral, cabe aos entes federados subnacionais a adequação nos seus territórios das ações nos moldes previstos pelas normas editadas pelo ente federal, garantindo e informando o cumprimento das metas. Vale ressaltar que, no custeio dos equipamentos de maior complexidade tecnológica, como as Unidades de Terapia Intensiva (UTI) adulto e infantil e das Unidades de Cuidado Intermediários ( $\mathrm{UCl}$ ) neonatal, os entes subnacionais comprometem também seus próprios recursos, assumindo $20 \%$ das despesas (Quadro I).

$O$ financiamento da Rede de Atenção à Urgência (RAU) também se caracteriza por voltar-se principalmente às ações específicas e especializadas. Haja vista os sete componentes que compõem a RAU, apenas o de Promoção, Prevenção e Vigilância à Saúde e o da AB não apresentam financiamento específico, enquanto em relação aos demais - Serviço Móvel de Atenção às Urgências (SAMU), Sala de Estabilização (SE), UPA24h, Hospitalar, e Atenção Domiciliar (AD) - apenas o último se refere ao cuidado mais abrangente, que inclui a prevenção, promoção, cura e reabilitação (Quadro II).

Conforme observado no Quadro II, são diversas as modalidades de financiamento e contrapartidas dos componentes que compõem a RAU. Em relação ao SAMU e sua Central de Regulação das Urgências, enquanto os recursos em investimentos são totalmente financiados pelo ente federal, tendo como contrapartida dos entes subnacionais a gestão e adequação técnica do sistema de operação, o custeio é compartilhado de forma tripartite. O valor do financiamento da SE, tanto para investimento como para custeio, é fixo, sendo de responsabilidade dos entes subnacionais os valores que excedam o estipulado.

Vale ressaltar que os recursos para investimento no componente UPA 24h, assim como ocorre no SAMU, são totalmente financiados pelo ente federal, sendo o custeio compartilhado de forma tripartite. O que chama atenção no componente UPA 24h, são os valores previstos para investimento, que podem chegar a 3 milhões de reais, e de custeio, que podem chegar a 500 mil mensais (Quadro II).

Em relação ao componente hospitalar, no qual também estão previstos grandes montantes para investimento e custeio, os valores para o financiamento das Portas de Entrada Hospitalares de Urgência e dos leitos de retaguarda que excederem o estipulado pela política são de responsabilidade dos entes subnacionais. $O$ financiamento para o Programa SOS Emergências, que tem como objetivo reduzir o tempo de espera e a superlotação dos grandes pronto socorros do País, prevê o assessoramento técnico das equipes dos hospitais. Assim, embora o recurso para o investimento e o custeio no âmbito desse programa seja transferido para os fundos dos entes subnacionais, os projetos são elaborados pelos estabelecimentos hospitalares.

Em relação ao componente Atenção Domiciliar, a Portaria nº 963 de 27 de maio de 2013 explicita como objetivo a reorganização do "processo de trabalho das equipes que prestam cuidado domiciliar na Atenção básica, ambulatorial e nos serviços de urgência e emergência e hospitalar, com vistas à redução da demanda por atendimento hospitalar e/ou redução do período de permanência de usuários internados, a humanização da atenção, a desinstitucionalização e a ampliação da autonomia dos usuários" (18). Esse recurso é destinado ao bloco de financiamento da AB e se refere ao custeio das equipes profissionais.

Dos seis componentes que compõem a Rede de Atenção Psicossocial (RAPS) apenas o da Atenção de Urgência e Emergência não apresenta financiamento especificado na portaria. A equipe Consultório de Rua (eCR), que integra o componente da Atenção básica tem financiamento especificado e transferido pelo bloco de financiamento da $A B$. A contrapartida municipal é a disponibilização de veículo para o deslocamento da equipe (Quadro III).

Os demais componentes da RAPS - Atenção Psicossocial Estratégica, Atenção Residencial de Caráter Transitório, Atenção Hospitalar, Estratégia de Desinstitucionalização e Estratégia de Reabilitação Psicossocial - tem financiamento previsto pelo bloco de financiamento da MAC. Vale ressaltar que parte do cuidado da RAPS financiado pelos recursos direcionados ao Bloco MAC se refere a atividades que funcionam de forma articulada entre a $A B$ e os Centros de Atenção Psicossocial (CAPS), como as Unidades de Acolhimento e os Serviços Residenciais Terapêuticos (SRT), que por sua natureza não se caracterizam como atividades tipicamente de média e alta complexidade. Em relação ao SRT, cabe destacar que o custeio previsto não é destinado aos módulos residenciais, mas a grupo de moradores. 
Merece menção ainda o componente Reabilitação Psicossocial, constituído de iniciativas de geração de trabalho e renda, empreendimentos solidários e cooperativas sociais, sendo os recursos destinados a programas de reabilitação psicossocial que beneficiam grupos de usuários do SUS. Devido a essas características, a portaria que normatiza o incentivo financeiro para esse componente não especifica controles nem contrapartidas.

Dos três componentes que conformam a Rede Viver sem Limites, a Atenção básica e a Atenção Hospitalar e de Urgência e Emergência não apresentam financiamento específico. As portarias que normatizam essa rede preveem financiamento para os Centros Especializados em Reabilitação (CER), para as Oficinas Ortopédicas e, um acréscimo de $20 \%$ no custeio dos Centros de Especialidades Odontológicas (CEO). Estes são os equipamentos que conformam o componente Atenção Especializada em Reabilitação Auditiva, Física, Intelectual, Visual, Ostomia e em Múltiplas Deficiências da Rede Viver sem Limites (Quadro IV).

De forma geral, a leitura das portarias que normatizam o financiamento das redes temáticas evidenciam três aspectos que valem ser ressaltados. O primeiro é que grande parte dos recursos são direcionados ao cuidado mais especializado, sendo financiados sobretudo pelo bloco de financiamento da MAC. Apenas em duas portarias, na que normatiza o componente "atenção domiciliar" da RAU e na que normatiza os incentivos para o Consultório de Rua (eCR) no componente "Atenção básica" da RAPS, os recursos estão vinculados especificamente ao bloco de financiamento da AB. Assim, mesmo que a Atenção básica seja considerada a organizadora das redes temáticas, o financiamento dessas políticas não privilegia esse nível de atenção à saúde.

Vale ressaltar que a atenção mais especializada, que exige maior volume de recursos financeiros, como aquelas que compõem os componentes financiados pelo bloco MAC, na sua maioria, apresenta configurações produtivas que exigem economia de escala. Por esse motivo, é possível afirmar que esse tipo de financiamento tende a se concentrar em municípios que apresentam maior centralidade, ou seja, com concentração populacional e de oferta de serviços complementares, facilidade de acesso de usuários residentes nos demais municípios e de mercadorias.

O segundo aspecto é aquele que evidencia a necessidade da realização de grandes esforços por parte dos entes subnacionais para a viabilização de parte considerável dos recursos ofertados. Não obstante os esforços empregados na construção dos planos regionais, dos diagnósticos de situação de saúde, dos mapas da oferta assistencial, entre outros, dependendo do tipo de financiamento pleiteado, será necessário o cumprimento de diversos outros requisitos. Merecem menção aqueles exigidos na elaboração de projetos para o investimento em novos equipamentos ou ampliação dos já existentes. Tais projetos são orientados por especificações técnicas bastante detalhadas e exigem a organização de grande quantidade de informações, bem como o envolvimento de profissionais qualificados. Além disso, após a efetivação do investimento, exige-se produção de relatórios e preenchimento dos sistemas de informação para monitoramento e avaliação, sujeitos às auditorias e verificações específicas do Ministério da Saúde, além dos controles gerais aos quais o uso dos recursos públicos já se encontra submetido. Dessa forma, a grande necessidade de mobilização de recursos técnicos e humanos para o cumprimento dos requisitos solicitados na adesão à rede, sobretudo no que diz respeito aos componentes cujos incentivos são maiores, privilegia os entes subnacionais com estruturas burocráticas e financeiras mais favorecidas. Em relação aos municípios, pode-se inferir que se trata daqueles localizados em regiões de maior dinâmica econômica, inseridos em espaços privilegiados de comando do território.

O terceiro aspecto é aquele que explicita a enorme fragmentação nos repasses dos recursos federais para os entes subnacionais. Além de observarmos a fragmentação dos recursos por rede temática, eles são ainda subdivididos entre os diferentes componentes que conformam cada rede, e que, por sua vez, são fragmentados em diferentes incentivos, seja para investimento, seja para custeio. Essa forma de repasse contraria a transferência de recursos de forma mais global, prejudicando a autonomia dos entes subnacionais, sobretudo o municipal.

Tal fragmentação não apenas restringe a autonomia dos entes subnacionais, sobretudo o municipal, em determinar o modelo assistencial mais adequado ao seu território, haja vista as necessidades de saúde local, mas, ao contrário, os submete a regras especificadas para cada componente, junto com seus padrões técnicos e de qualidade, suas lógicas produtivas.

\section{CONCLUSÃO}

Pode-se concluir que o financiamento das redes temáticas, além de favorecer o modelo médico assistencial por meio da transferência de recurso prioritariamente para a atenção à saúde voltada à média e alta complexidade, parece tender às regiões já privilegiadas como espaços de atração de investimentos capitalistas, não apenas porque o acesso aos recursos, em grande parte, exige capacidade burocrática e financeira previamente existente, mas também porque são ações e serviços que dependem de economia de escala. 

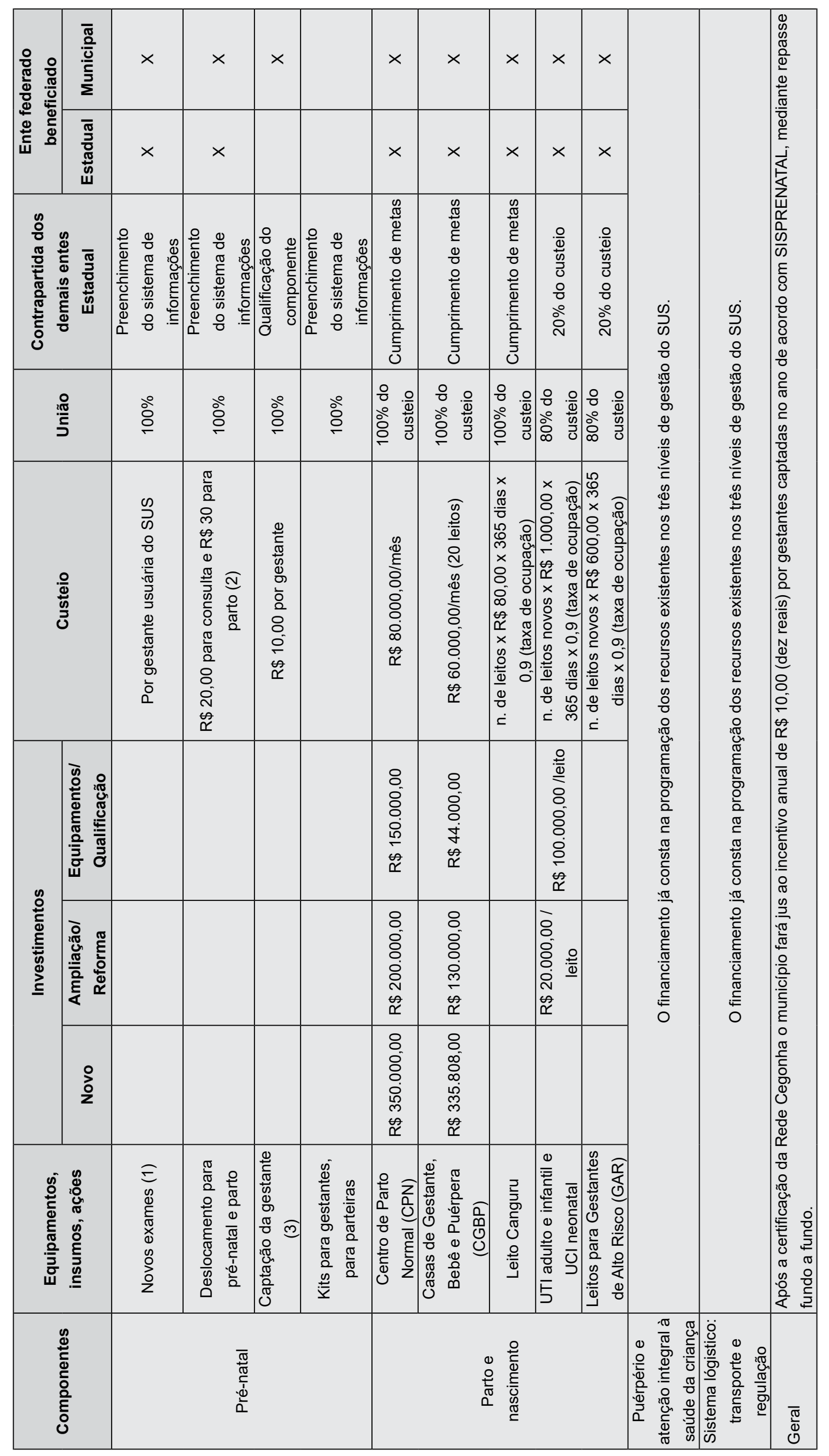

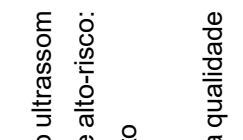

인

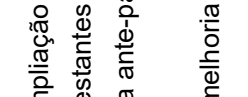

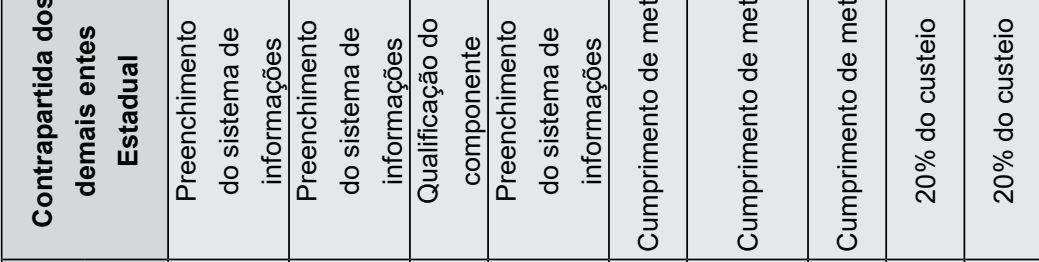

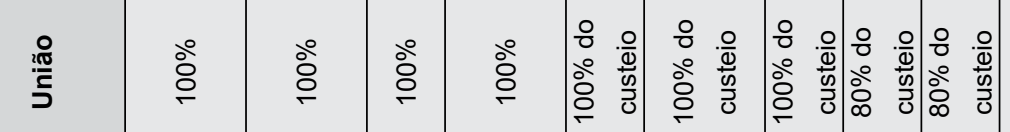

\begin{tabular}{|c|c|c|c|c|c|c|c|}
\hline \multirow[b]{2}{*}{ 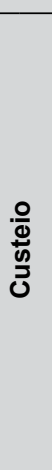 } & \multirow[b]{2}{*}{ 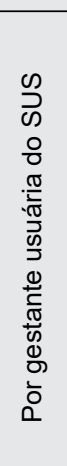 } & \multirow[b]{2}{*}{ 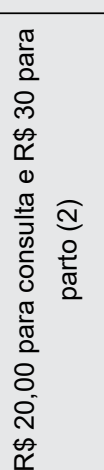 } & \multirow[b]{2}{*}{ 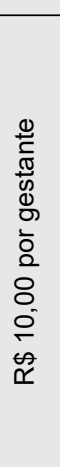 } & & & & \\
\hline & & & & 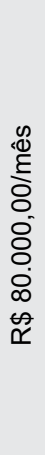 & 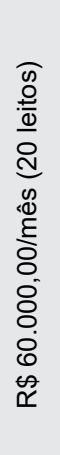 & 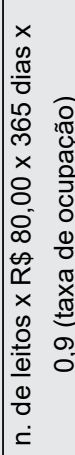 & 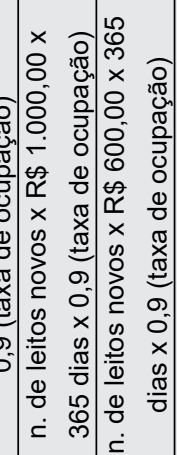 \\
\hline
\end{tabular}

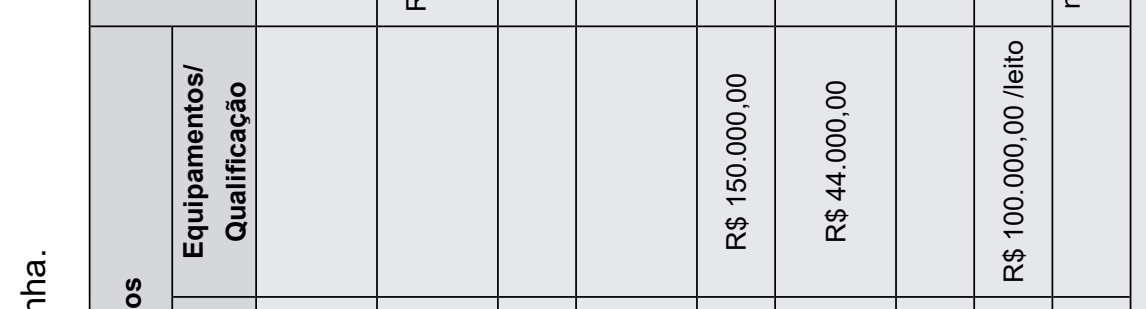

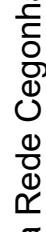

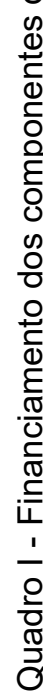
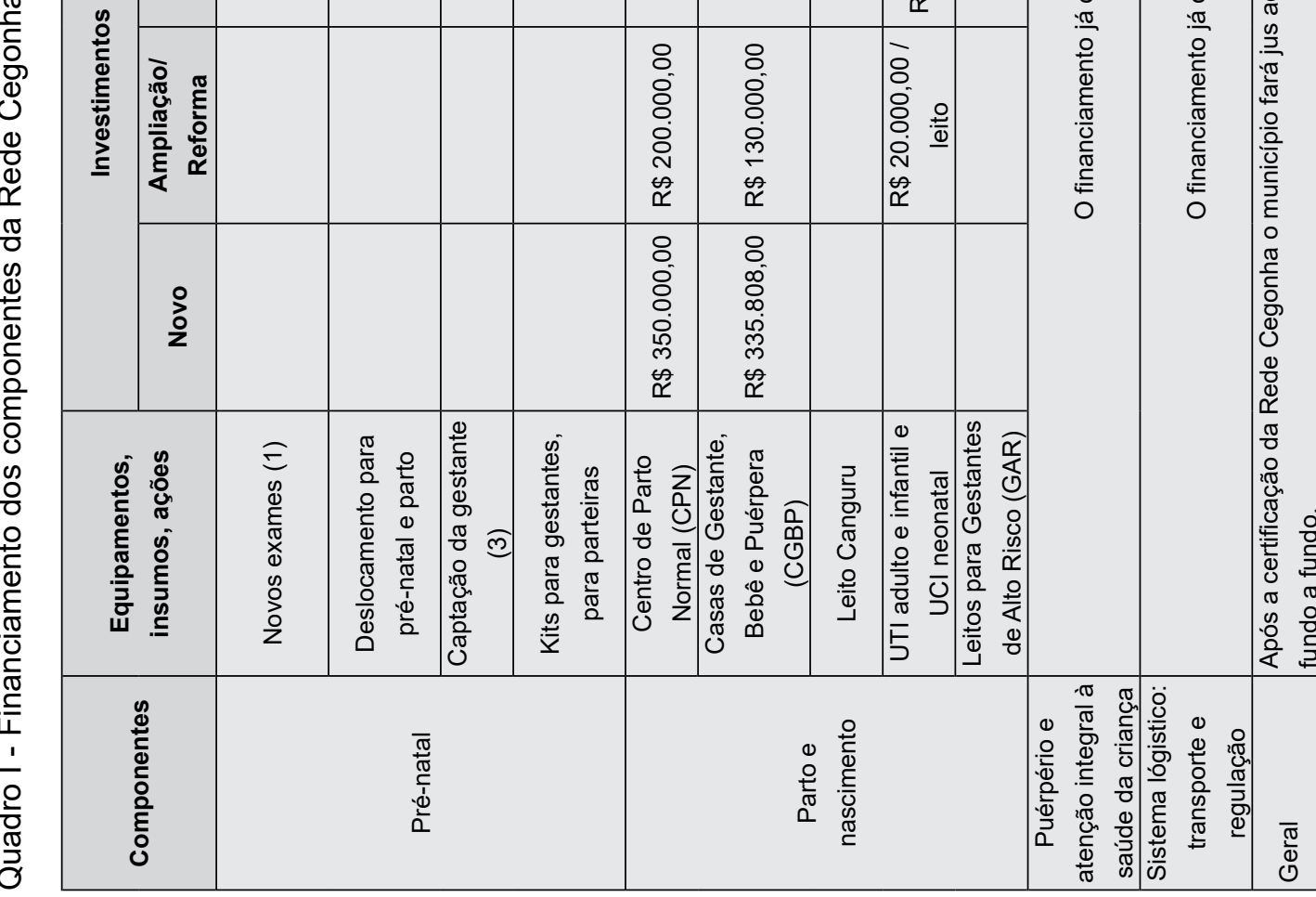

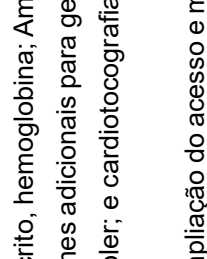

造

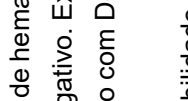

ब

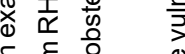

है

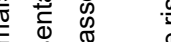

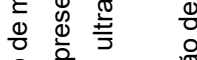

잉

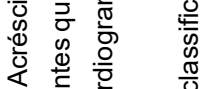

के

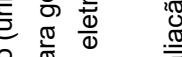

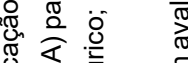

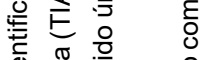

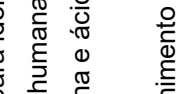

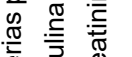

응

范

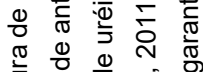

章

0.

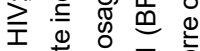

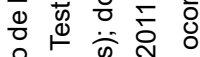

음

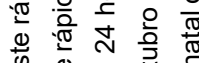

幽

is 过

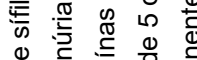

范

응응 흥유

is

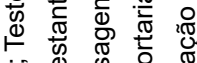

बं

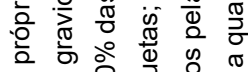
造

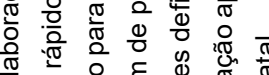
西

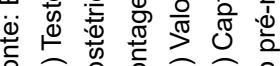


Regionalização e financiamento do SUS

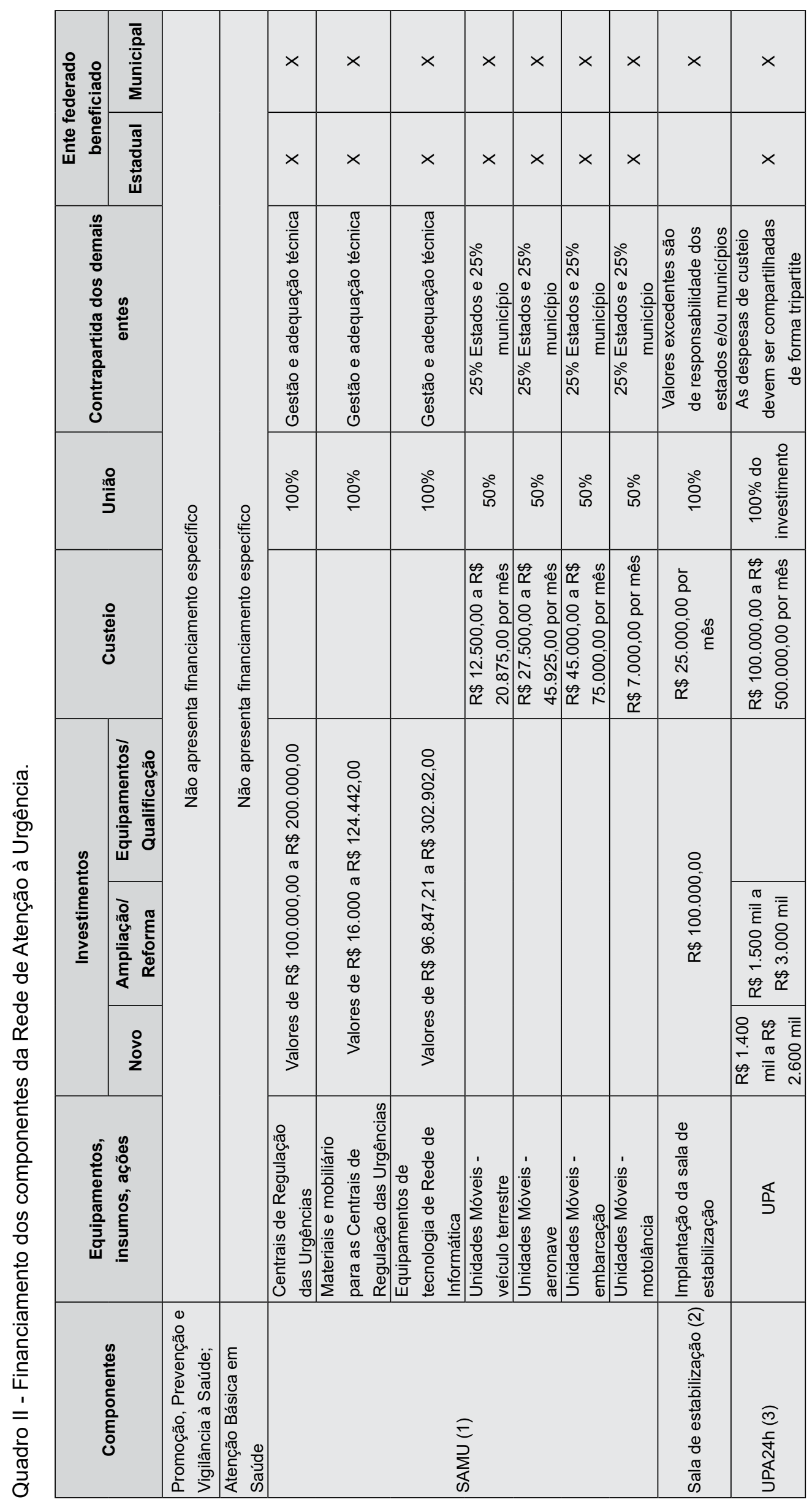




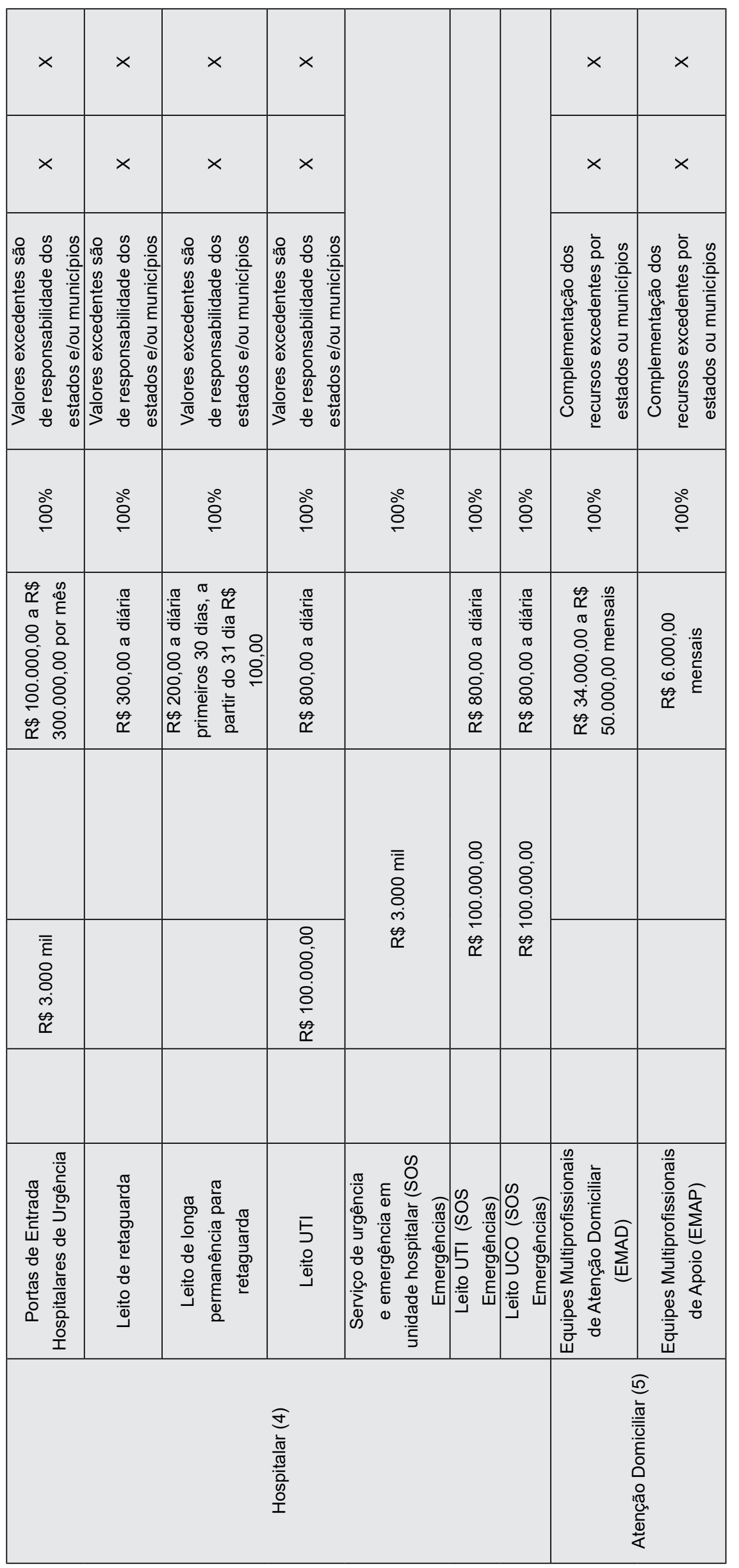

容 它竞 范芯 ल्र 啇 융 용 ฮ 00 ه + 용 2. ल.

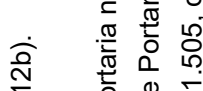
㲾

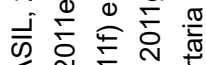

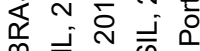
क्ष क जि

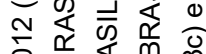

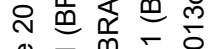

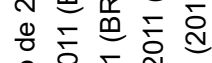
일 응 응 융 율 융

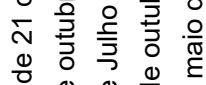

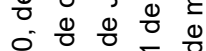
ه 응

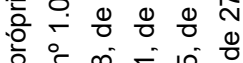
둥 일

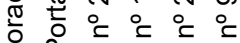

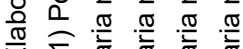
ए क्ष

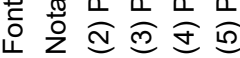


Regionalização e financiamento do SUS

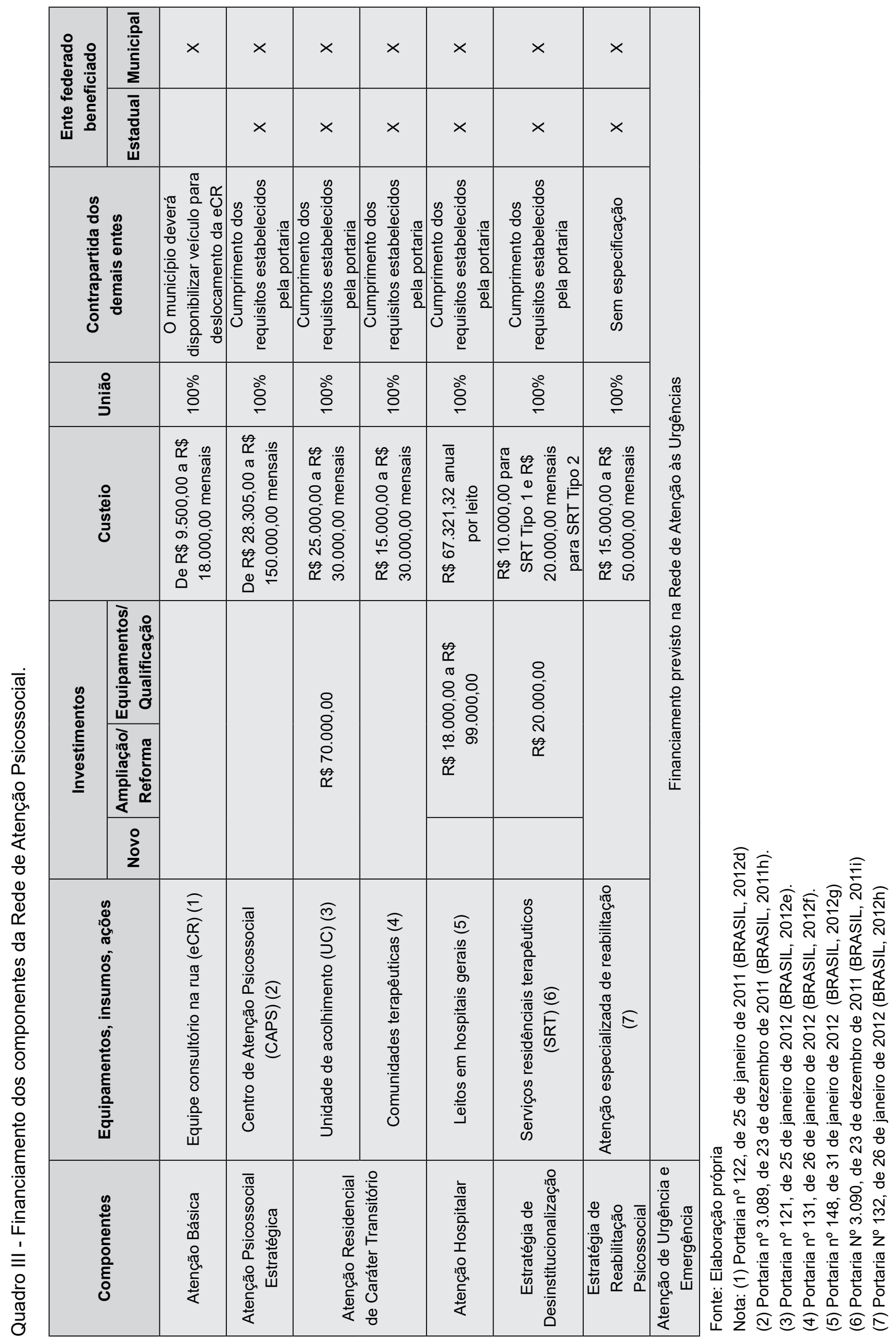




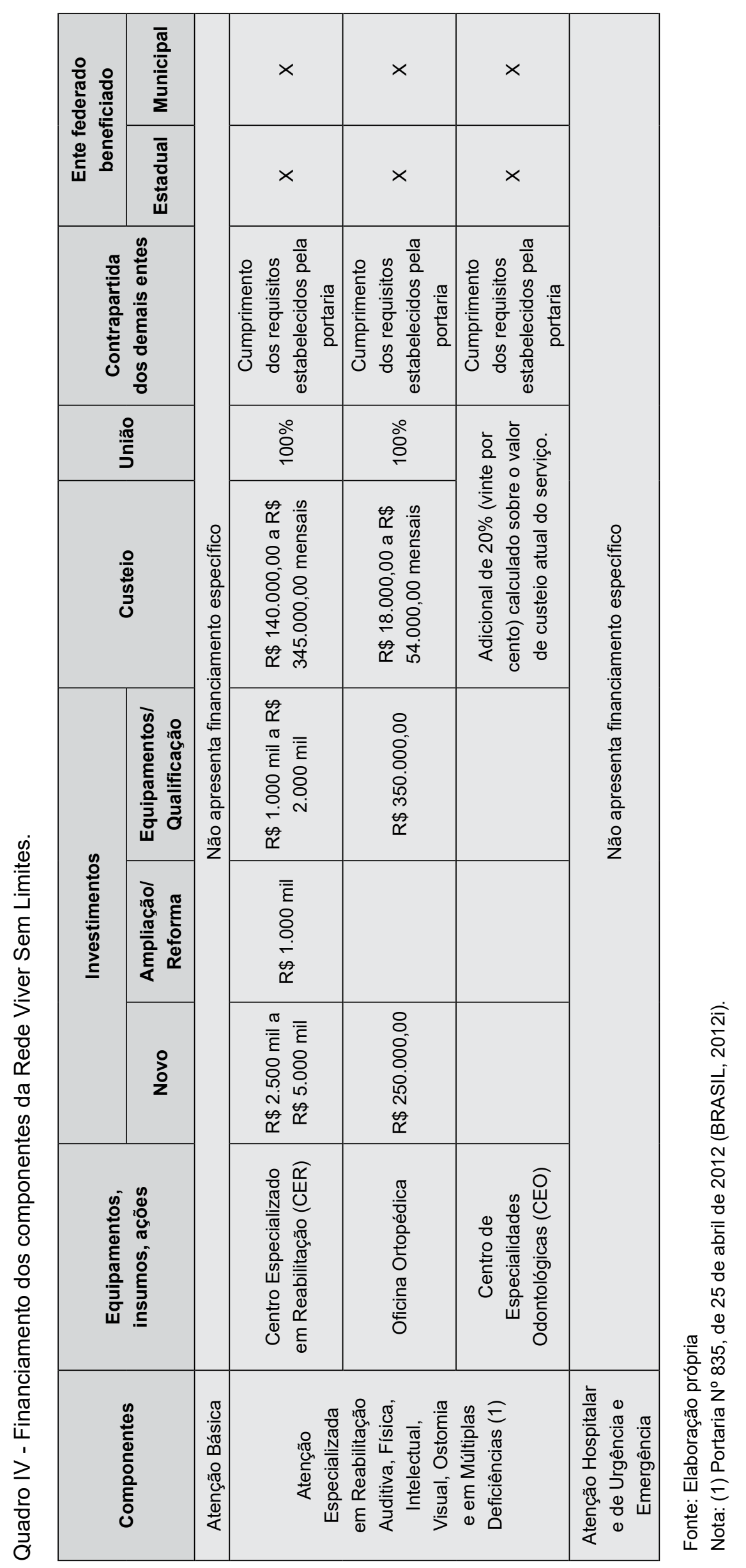




\section{REFERÊNCIAS}

1. Mendes AN. Tempos turbulentos na saúde pública brasileira: impasses do financiamento no capitalismo financeiro. São Paulo: Hucitec; 2012.

2. Brasil. Lei n. 8.080 , de 19 de setembro de 1990. Dispõe sobre as condições para a promoção, proteção e recuperação da saúde. Diário Oficial da União; Brasília; 20 Set. 1990; Seção1.

3. Duarte LS. Desenvolvimento desigual e a regionalização do SUS: uma análise territorial dos recursos financeiros para as redes de atenção à saúde no Estado de São Paulo (2009-2014) [tese]. São Paulo: Universidade de São Paulo; 2016.

4. Mello JMC. O Capitalismo Tardio. São Paulo: Brasiliense; 1982.

5. Brandão CA. Território e desenvolvimento: as múltiplas escalas entre o local e o global. Campinas: Editora da Unicamp; 2007.

6. Pochmann M. Desigualdades econômicas no Brasil. São Paulo: Ideias \& Letras; 2015.

7. Ministério do Planejamento, Desenvolvimento e Gestão (BR), Instituto Brasileiro de Geografia e Estatística. Produto interno bruto dos municípios: 2010-2015. Rio de Janeiro: IBGE; 2017.

8. Contel FB, Balbim R. Região e regionalização: subsídios teóricos para o ordenamento territorial e o desenvolvimento brasileiro. In: Boueri R, Costa MA, organizadores. Brasil em desenvolvimento 2013: Estado, Planejamento e Políticas Públicas. Brasília: IPEA; 2013. p. 25-47. v. I.

9. Brasil. Ministério da Saúde. Portaria n. 4.279, de 30 de dezembro de 2010. Estabelece diretrizes para a organização da Rede de Atenção à saúde no âmbito. Diário Oficial da República Federativa do Brasil, Brasília. 2010.

10. Brasil. Decreto n. 7.508, de 28 de junho de 2001. Regulamentação da Lei $n^{\circ} 8.080 / 90$. Brasília: Ministério da Saúde; 2011. 16 p. (Série E. Legislação de Saúde).

11. Brasil. Ministério da Saúde. Portaria n. 1.473, de 24 de junho de 2011. Institui os Comitês Gestores, Grupos Executivos, Grupos Transversais e os Comitês de Mobilização Social e de Especialistas dos compromissos prioritários de governo organizados por meio de Redes Temáticas de Atenção à Saúde. Diário Oficial da República Federativa do Brasil, Brasília. 2011.

12. Magalhães HM Jr. Redes de atenção à saúde: rumo à integralidade. Divulg Saúde Debate. 2014;52:15-37.

13. Brasil. Ministério da Saúde. Portaria n. 1.459, de 24 de junho de 2011. Institui, no âmbito do Sistema Único de Saúde - SUS - a Rede Cegonha. Diário Oficial da República Federativa do Brasil, Brasília. 2011.

14. Brasil. Ministério da Saúde. Portaria n. 1.600, de 7 de julho de 2011. Reformula a Política Nacional de Atenção às Urgências e institui a Rede de Atenção às Urgências no SUS. Diário Oficial da República Federativa do Brasil, Brasília. 2011.

15. Brasil. Ministério da Saúde. Portaria n. 3.088, de 23 de dezembro de 2011. Institui a Rede de Atenção Psicossocial para pessoas com sofrimento ou transtorno mental e com necessidades decorrentes do uso de crack, álcool e outras drogas, no âmbito do Sistema Único de Saúde. Diário Oficial da República Federativa do Brasil, Brasília. 2011.

16. Brasil. Ministério da Saúde. Portaria n. 793, de 24 de abril de 2012. Institui a Rede de Cuidados à Pessoa com Deficiência no âmbito do Sistema Único de Saúde (SUS). Diário Oficial da República Federativa do Brasil, Brasília. 2012.

17. Brasil. Ministério da Saúde. Portaria n. 874, de 16 de maio de 2013. Institui a Política Nacional para a Prevenção e Controle do Câncer na Rede de Atenção à Saúde das Pessoas com Doenças Crônicas no âmbito do Sistema Único de Saúde (SUS). Diário Oficial da República Federativa do Brasil, Brasília. 2013.

18. Brasil. Ministério da Saúde. Portaria n. 963, de 27 de maio de 2013. Redefine a Atenção Domiciliar no âmbito do Sistema Único de Saúde (SUS). Diário Oficial da República Federativa do Brasil, Brasília. 2013. 
Endereço para correspondência:

Ligia Schiavon Duarte

Instituto de Saúde da Secretaria de Estado da Saúde de São Paulo

Rua Santo Antônio, 590

Bairro: Bela Vista

CEP: 01033-000 - São Paulo - SP - Brasil

E-mail: ligia.duarte@uol.com.br 